\title{
Mechanisms of Action of Zishen Yutai pills in Treating Premature Ovarian Failure Determined by Integrating UHPLC-Q-TOF-MS and Network Pharmacology Analysis
}

\section{Lei Dang}

Huazhong University of Science and Technology

Chunbo Zhang

Post-Doctoral Research Center of Guangzhou Pharmaceutical Holdings Ltd

\section{Biru Su}

Guangzhou Baiyunshan Zhongyi Pharmaceutical Co. Ltd

\section{Ning $\mathrm{Na}$}

Guangzhou Baiyunshan Zhongyi Pharmaceutical Co. Ltd

\section{Qiuling Huang}

Guangzhou Baiyunshan Zhongyi Pharmaceutical Co. Ltd

\section{Su Zhou}

Huazhong University of Science and Technology

\section{Meng Wu}

Huazhong University of Science and Technology

\section{Wenqing $\mathrm{Ma}$}

Huazhong University of Science and Technology

\section{Man Wang}

Huazhong University of Science and Technology

\section{Pengfei Cui}

Huazhong University of Science and Technology

\section{Yan Li}

Huazhong University of Science and Technology

Shixuan Wang ( $\nabla$ shixuanwang@tjh.tjmu.edu.cn )

Huazhong University of Science and Technology

\section{Research Article}

Keywords: Zishen Yutai pills, premature ovarian failure, active component, network pharmacology, molecular mechanism, therapeutic mechanism 
Posted Date: January 6th, 2022

DOI: https://doi.org/10.21203/rs.3.rs-1176592/v1

License: (c) (i) This work is licensed under a Creative Commons Attribution 4.0 International License. Read Full License 


\section{Abstract}

Background: Zishen Yutai (ZSYT) pill, a patent Chinese medicine, has been widely used in the treatment of infertility, abortion, and adjunctive treatment of in vitro fertilization (IVF) for decades. Recently, the results of clinical observations showed that premature ovarian failure (POF) patients exhibited improved expression of steroids and clinical symptoms associated with hormone disorders after treatment with ZSYT pills. However, the pharmacological mechanism of action of these pills remains unclear.

Methods: The components of ZSYT found in blood circulation were identified via ultra-high performance liquid chromatography-quadrupole time-of-flight mass spectrometry (UHPLC-Q-TOF-MS) technique in the serum of POF mice after oral administration of ZSYT pills. The potential targets of components were screened using Traditional Chinese Medicine Systems Pharmacology Database, Traditional Chinese Medicine Database@Taiwan, Drugbank Database, PubChem, HIT, Pharmapper, and SwissTargetPrediction. The target genes associated with POF were collected from Online Mendelian Inheritance in Man Database, PharmGkb, Genecards, therapeutic target database, and Genetic Association Database. The overlapping genes between the potential targets of ZSYT components and the target genes associated with POF were clarified via protein-protein interaction (PPI), pathway, and network analysis.

Results: Nineteen components in ZSYT pills were detected in the serum of POF mice after oral administration. A total of 695 ZSYT-related targets was screened, and 344 POF-related targets were collected. From the results of ZSYT-POF PPI analysis, CYP19A1, AKR1C3, ESR1, AR, and SRD5A2 were identified as key targets via network analysis, indicating their core role in the treatment of POF with ZSYT pills. Moreover, the pathway enrichment results suggested that ZSYT pills treat POF primarily by regulating neuroactive ligand-receptor interaction, steroid hormone biosynthesis, and ovarian steroidogenesis.

Conclusions: We demonstrated that regulation of neuroactive ligand-receptor interaction, steroid hormone biosynthesis, and ovarian steroidogenesis are very likely to be therapeutic mechanism of ZSYT pills in treating POF. Our study suggests that combining the analysis of ZSYT pills components in blood in vivo in the POF models and network pharmacology prediction may offer a tool to characterize the mechanism of ZSYT pills in the POF.

\section{Background}

Premature ovarian failure (POF) is an ovarian dysfunction with cessation of menstruation before 40 years of age (1). POF probably occurs when the number of ovarian follicles is exhausted before the typical age of physiological menopause $(2,3)$.

The pathological causes of POF include genetic, autoimmune, therapeutic, and environmental stimulus, etc. (4). The prevalence of POF in women is approximately $1 \%$ (5), and it occurs in women between 20 and 40 years of age (6). 
The clinical diagnostic criteria of POF are amenorrhea together with elevated gonadotropin levels (folliclestimulating hormone $[\mathrm{FSH}]>20 \mathrm{IU} / \mathrm{L}$ ), low estradiol (E2) levels $(<20 \mathrm{pg} / \mathrm{mL})$, and low anti-Müllerian hormone (AMH) levels $-<0.5 \mathrm{ng} / \mathrm{mL}(<1 \mathrm{ng} / \mathrm{mL})$ (7). POF affects the quality of life of women by causing hot flushes, excessive sweating, hair loss, skin and mucous membrane dryness, and even loss of fertility that is devastating for women of reproductive age (1). In the long term, women with POF face an increased risk of morbidity, developing conditions such as osteoporosis, cardiovascular diseases, and type 2 diabetes. Because of the complexity of POF pathogenesis, the optimal remedy for the treatment of POF is still under investigation (4).

Traditional Chinese medicine (TCM) has been widely used in treating gynecological disorders in Asian countries and has been proposed for treating POF (8). Zishen Yutai (ZSYT) pills, a Chinese patent medicine, have been clinically used to treat infertility, abortion, and adjunctive treatment of in vitro fertilization (IVF) $(9,10)$. Recent studies with ZSYT pills identified improvements in clinical symptoms of hormone disorders and an increase in the FSH and luteinizing hormone (LH) levels in the treatment group compared to the controls, indicating the clinical potential of ZSYT in treating POF $(11,12)$. Although the methanol extracts of ZSYT pills were analyzed previously in vitro via UHPLC-Q-TOF-MS (13), the active components in vivo and their pharmacological targets in treating POF have not been elucidated. Our study indicates synergistic effects of ZSYT components and suggests their pharmacological mechanisms in treating POF.

ZSYT pills contain 15 herbal plant materials. Thousands of components from the ZSYT pill are absorbed through the digestive system and distributed into internal organs by blood circulation. These absorbed components are likely to have therapeutic effects (14).

In this study, the UHPLC-Q-TOF-MS analysis approach was used to identify the absorbed components of the ZSYT pills in the POF mouse model in vivo. Next, network pharmacology analysis was performed to screen potential active ingredients, seek their targets for treating POF, and investigate the underlying pharmacological mechanisms. The combination of UHPLC-Q-TOF-MS and network pharmacology help to build the pharmacology network for exploring the therapeutic mechanism of ZSYT pills in the treatment of POF, and may lead to a full understanding of the interactions taking place between absorbed components of ZSYT pills in vivo and potential targets involved in the POF treatment (Figure 1).

\section{Methods}

Preparation of ZSYT Pills

Fifteen herbs, Semen Cuscutae, Fructus Amomi Villosi, Radix Rehmanniae Preparata, Radix Ginseng, Herba Taxilli, Equus asinus L., Radix Polygoni Multiflori, Folium Artemisiae Argyi, Radix Morindae Officinalis, Rhizoma Atractylodis Macrocephalae, Radix Codonopsis, Cornu Cervi Degelatinatum, Fructus Lycii, Radix Dipsaci, and Cortex Eucommiae, comprising the ZSYT pills (batch No. A00010) were provided by Guangzhou Baiyunshan Zhongyi Pharmaceutical Co., Ltd. The main ingredient of Cornu Cervi 
Degelatinatum is $\mathrm{Ca}_{3}\left(\mathrm{PO}_{4}\right)_{2}$ and it has no botanical name (15). Botanical materials were authenticated before manufacturing in good manufacturing practice-certified pharmaceutical factories. The preparation and quality control of the ZSYT pills were performed according to the Chinese Pharmacopeia (2020 edition) and the Ministry of Public Health of the People's Republic of China - TCM prescription preparation (Volume 16, standard number: WS $_{3}-$ B-3113-98). For the experiments, ZSYT pills were broken into powder, passed through an 80-mesh screen, and stirred uniformly in pilot facilities.

Animals and Treatment

Female C57BL/ 6 mice between 6 and 8 weeks of age were obtained from the HUNAN SJA Laboratories Animal Co., Ltd. The mice were housed in a temperature-controlled environment $\left(22 \pm 2{ }^{\circ} \mathrm{C}\right)$ under a $12 / 12 \mathrm{~h}$ light/dark cycle with a relative humidity of $60 \%$. The mice were separated into five mice per cage for seven days to adapt to the environment. All mice were provided free access to chow and tap water.

After one-week acclimatization, 20 C57BL/ 6 female mice were randomly divided into two groups: POF and POF+ZSYT, with 10 mice in each group. Mice in the POF and POF+ZSYT groups were treated with cisplatin to induce POF. The mice were intraperitoneally administered $2 \mathrm{mg} / \mathrm{kg}$ cisplatin (Sigma Aldrich, USA) once a day for 10 days $(16,17)$. Cisplatin was freshly prepared in saline immediately before use.

After intraperitoneal administration for 10 days, the mice in the POF+ZSYT group were orally administered ZSYT pills ( $3.90 \mathrm{~g} \cdot \mathrm{kg}^{-1}$ body weight), whereass the mice in the POF group were orally administered an equal volume of $0.3 \%$ CMC-Na solution. The ZSYT pill powder was dissolved in $0.3 \%$ CMC-Na solution as an oral suspension. All mice were dosed twice daily for 3 days (18). All mice were euthanized to collect blood samples $1 \mathrm{~h}$ after the last dose.

The experiments and procedures were performed following the principles of laboratory animal use and care. The study was approved by the Institutional Animal Ethics Committee of the Guangzhou General Pharmaceutical Research Institute Co., Ltd.

Collection and Preparation of Serum Samples

Blood samples were collected from the abdominal aorta after euthanasia. Blood samples were left to stand at room temperature for $30 \mathrm{~min}$ and then centrifuged at 3,000 rpm for $10 \mathrm{~min}$ at $4^{\circ} \mathrm{C}$ to obtain serum. The serum supernatants were collected and mixed at equal volumes before storage at $-80^{\circ} \mathrm{C}$. Next, the $100 \mu \mathrm{L}$ of the serum mixture was mixed with $400 \mu \mathrm{L}$ of methanol, whirled for 3 min, and then centrifuged at $13,000 \mathrm{rpm}$ for $10 \mathrm{~min}$ at $4^{\circ} \mathrm{C}$. The $300 \mu \mathrm{L}$ of supernatant was transferred to a clean tube and dried under a gentle flow of nitrogen gas at $30^{\circ} \mathrm{C}$. The residue was dissolved in $75 \mu \mathrm{L}$ of methanol and centrifuged at $13000 \mathrm{rpm}$ for $10 \mathrm{~min}$ at $4^{\circ} \mathrm{C}(19)$. The supernatant was collected, and $4 \mu \mathrm{L}$ was injected for UHPLC-Q-TOF-MS analysis.

Chromatography Conditions and UHPLC-Q-TOF-MS Analysis 
The UHPLC chromatographic qualitative analysis was performed on a SCIEX X500R QTOF LC-MS/MS system (AB SCIEX Pte Ltd., USA), equipped with an ExionLC degasser, an ExionLC AD pump, an ExionLC AD autosampler, an ExionLC AD column oven, and an ExionLC PDA detector.

A Hypersil gold C18 $(150 \mathrm{~mm} \times 2.1 \mathrm{~mm}, 1.9 \mathrm{~m}$, Thermo Fisher) column was used to separate the extract components. Mobile phase A consisted of $0.1 \%$ formic acid $(\mathrm{v} / \mathrm{v})$ solution, and mobile phase $\mathrm{B}$ was acetonitrile. The flow rate was $0.4 \mathrm{~mL} \mathrm{~min}^{-1}$ and the column temperature was maintained at $40^{\circ} \mathrm{C}$. The gradient program was set as follows: 0-10 min, $5 \%$ B; $10-20$ min, 5-10\% B; $20-40$ min, $10-25 \%$; $40-60$ min, 25-30\% B; 60-75 min, 30-40\% B; 75-90 min, 40-75\% B; 90-120 min, 75-90\% B; 120-140 min, 90-100\% B; 140-190 min, 100\% B (13). Data acquisition and analysis were carried out using Sciex PeakView ${ }^{\mathrm{TM}} 1.7$ software.

Mass Spectrometry

Mass spectrometry of serum samples was conducted on a SCIEX X500R QTOF mass spectrometer (AB SCIEX Pte Ltd., USA) equipped with an electrospray ionization (ESI) source connected to the UPLC system. Sciex PeakView ${ }^{\text {TM }}$ 1.7. software (AB SCIEX Pte Ltd., USA) was used for data acquisition and processing.

The analysis was performed in both positive and negative ESI ion modes. The information-dependent acquisition scan mode was selected, and the mass range was recorded at m/z 100-1500 (13). Calibration solutions for the SCIEX X500 System were used to calibrate the mass spectrometer in the positive and negative ion modes.

Data Processing

After data acquisition via UHPLC-Q-TOF-MS, components simultaneously with the same mass and equal fragment intensity between the results from the POF+ZSYT and POF groups were excluded. Next, the mass and fragments of the remaining candidate components were matched with our previously detected components from methanol extract in ZSYT pills (13) and online databases including the Chemspider (http://www.chemspider.com/), SCIEX-Standard Chinese medicine Mass Spectral Database, SCIEX metabolite Mass Spectral Database, SCIEX lipids Mass Spectral Database (19), and Sciex PeakView ${ }^{\mathrm{TM}}$ 1.7. (AB SCIEX Pte Ltd., USA) (20). Thereafter, the ZSYT pill components found in the POF mouse in vivo were identified.

Target Collection

The relevant targets of the ZSYT pills components identified in vivo were obtained from the Traditional Chinese Medicine Systems Pharmacology Database (TCMSP, http://lsp.nwu.edu.cn/tcmsp.php), TCM Database@Taiwan (http://tcm.cmu.edu.tw/), Drugbank Database (https://www.drugbank.ca/), PubChem (https://pubchem.ncbi.nlm.nih.gov/), HIT (lifecenter. sgst. cn/hit /), Pharmapper (http://www.lilabecust.cn/pharmmapper/), and Swiss target prediction (http://www.swisstargetprediction.ch/) (21). By 
inputting all molecular formulae of the absorbed components into these databases, we obtained information on their potential targets; sufficient targets associated with the absorbed components were obtained.

The target genes associated with POF were collected from the Online Mendelian Inheritance in Man Database (OMIM, http://www.omim.org/), Pharmacogenomics Knowledge Base (PharmGkb, https://www.pharmgkb.org/), Genecards (http://www.genecards.org/), therapeutic target database (TTD, https://db.idrblab.org/ttd/), and Genetic Association Database (GAD, https://geneticassociationdb.nih.gov/).

POF-related targets overlapping with the absorbed component-relevant targets of ZSYT pills were retained for network construction and analysis.

Network Analysis and Construction

To explore the ZSYT pill's mechanisms of action in the treatment of POF, we investigated the pathways associated with POF treatment. First, the STRING database (http://string-db.org) was used to filter possible protein-protein interactions (PPIs) (22). The attained PPIs with high confidence $>0.7$ were selected for the following analysis. Second, the shared targets between the ZSYT compounds in vivo and POF were constructed and visualized using Cytoscape v3.6.1. The ZSYT pills absorbed components and target genes were expressed as nodes, whereas the compounds and potential targets interactions were expressed as edges in the graphical networks. Thirdly, the Go enrichment analysis gene ontology (GO) and Kyoto Encyclopedia of Genes and Genomes (KEGG) enrichment were conducted using the functional annotation tool of DAVID Bioinformatics Resources 6.7 (http://david.abcc.ncifcri.gov/) and Bioinformatics (http://www.bioinformatics.com.cn/), respectively $(23,24)$.

\section{Results}

\section{Screening for the absorbed components of ZSYT pills in vivo}

The medicated serum samples were collected after the oral administration of ZSYT pills in the POF mice, while the blank serum samples were collected after the oral administration of $0.3 \%$ CMC-Na solution in the POF mice. The both samples were analyzed via UHPLC-Q-TOF-MS. The peaks of candidate components in the medicated serum samples were extracted from both positive and negative ion chromatograms after excluding the same mass with equal fragment intensity in the blank serum (Figure 2). The candidate components were identified qualitatively using Sciex PeakView ${ }^{\mathrm{TM}}$ 1.7. software (25).

The MS/MS spectra of all candidate components were evaluated and matched with hits in the Chemspider (http://www.chemspider.com/), SCIEX-Standard Chinese medicine Mass Spectral Database, SCIEX metabolite Mass Spectral Database, SCIEX lipids Mass Spectral Database, and our previously identified chemical profiles in ZSYT pills. 
A total of 19 components were successfully characterized by comparison with the online database and our previously identified components (Table S1). Six compounds were identified previously, and 13 compounds were newly identified.

Shared Targets Between Absorbed Component-related Targets of ZSYT In Vivo and POF-related Targets

Based on the results of UHPLC-Q-TOF-MS analysis, 19 ZSYT pills components absorbed in vivo were used to obtain the ZSYT-related targets. In this study, the 695 ZSYT-related targets were screened from the TCMSP, TCM Database@Taiwan, Drugbank Database, PubChem, HIT, Pharmapper, and Swiss target prediction corresponding to the 19 absorbed components in the ZSYT in vivo.

The POF-related target proteins were screened from OMIM, Pharmacogenomics Knowledge base (PharmGkb), GAD, Drugbank Database, TTD, and recently published papers. Only "Homo sapiens" proteins linked to POF were selected. We obtained a total of 344 genes.

Among all targets from ZSYT pills and POF, 56 overlapped genes were identified and retained for network construction (Table 1). The composed compound-target network consisted of 77 nodes (19 component target nodes and 183 component edges) and 258 edges (Figure 3).

Absorbed Components of ZSYT-POF PPI Network

A total of 56 overlapping genes were obtained by searching for the intersection of the targets of absorbed components from ZSYT pills in vivo and the 344 POF-related targets.

Among the overlapping genes, a total of 202 PPIs with high confidence (P-value $>0.7)$ were obtained and selected from STRING databases for PPI network construction using Cytoscape software (Table S2).

The absorbed components of the ZSYT-POF PPI network formed 45 nodes and 100 edges (Figure 4). The key targets were analyzed using central network evaluation. Twelve targets with 50 interactions were found with a degree greater than 6 (Table S3) after analysis via Cytohubba. Among the top-ranked hub targets, CYP19A1, aldo-keto reductase (AKR) 1C3, ESR1, AR, and SRD5A2 were identified as key targets via network analysis, indicating their core role in the treatment of POF by ZSYT pills.

Pathway Enrichment

GO enrichment analysis showed that the majority of these 56 targets were identified using the DAVID Bioinformatics Resources. There were 183 biological processes (BP), 24 cellular components (CC), and 62 molecular functions (MF) identified. The top 15 significantly enriched terms in the BP, CC, and MF categories $(P<0.05)$ are shown in Figure 5A-C. Specifically, most of these target proteins were enriched in responses to drugs, progesterone, androgen metabolic processes, chemical synaptic transmission, positive regulation of cell proliferation, and steroid and androgen biosynthetic processes, indicating multiple synergies of absorbed components from ZSYT pills with biological processes. It also revealed that the absorbed components from ZSYT pills might influence the steroid hormone receptor activity, drug binding, oxidoreductase activity, steroid and enzyme binding in the cellular membrane, intracellular 
membrane-bounded organelle, or endoplasmic reticulum membrane potentially exerting a therapeutic effect on POF.

Moreover, KEGG pathway enrichment analysis demonstrated that seven pathways $(P<0.05)$ were affected by the components absorbed from ZSYT pills in the in vivo POF model, namely steroid hormone biosynthesis, ovarian steroidogenesis, cocaine addiction, cytochrome P450, neuroactive ligand-receptor interaction, serotonergic synapse, and dopaminergic synapse. These pathways were associated with steroid hormone biosynthesis and metabolism (3 pathways), drug metabolism (2 pathways), and signal transduction (2 pathways) (Figure 6).

\section{Discussion}

ZSYT pill, a Chinese patent medicine, has been widely used for fertility and reproduction for decades (9, 10). Although multiple monomeric compounds in the ZSYT pills exert their therapeutic effects in improving reproduction, the combined activity especially of those absorbed into the blood circulation and their metabolites in vivo compounds is poorly understood. Recently, the ZSYT pills showed therapeutic effects on ovarian dysfunction such as diminished ovarian reserve, primary ovarian insufficiency, and polycystic ovary syndrome (26).

In ovarian dysfunction, POF patients struggle with the loss of gametogenic and steroidogenic functions of the ovary and low probability of pregnancy; it impacts approximately one in 100 women under the age of 40 years; there are currently no effective treatments available (27). In recent clinical studies that used ZSYT pills to treat POF patients, increased expression of FSH and $\mathrm{LH}$, and the improvement of hormone disorders clinical symptoms were observed $(12,13)$. However, the therapeutic mechanism of ZSYT pills in vivo remains obscure.

In this study, we first identified the absorbed components of ZSYT pills in POF mice via UHPLC-Q-TOF-MS and employed a network pharmacology-based prediction of the absorbed compound-related gene targets and gene-pathway interactions to interpret the therapeutic mechanism of ZSYT pills in treating POF.

A total of 19 components were identified in the POF mice after treatment with ZSYT pills compared to those treated with the solvent. Among these components, six ingredients were previously identified in methanol extracts of ZSYT pills and 13 ingredients were newly identified (13).

The network pharmacology analysis we developed based on the ZSYT pills chemical profile presented 695 hormone disorders candidate gene targets interacting with 19 potentially active ZSYT compounds. Combining 695 candidate targets and 344 POF-related genes, there were 56 overlapping gene targets of ZSYT pills components in the treatment of POF. In this ZSYT absorbed compounds-related gene targets, CYP19A1, AKR1C3, ESR1, AR, and SRD5A2 were selected as potential core genes with a higher degree of probability compared to other gene targets. 
CYP19A1 is the key steroidogenic enzyme that transforms androstenedione to estrone and testosterone to E2 in granulosa cells $(28,29)$. Inhibition of CYP19A1 could reduce the steroidogenic capacity of antral follicles (30). AKR1C3 is one of four members of the AKR superfamily among the $17 \beta$-hydroxysteroid

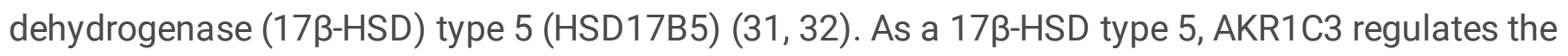
bioactivity and biosynthesis of steroids by the different extent of 3-keto-, 17-keto-, and 20-ketosteroid reduction (33). It also acts as a co-activator of AR in its moonlighting function (34).

ESR1 regulates steroidogenesis in theca cells and acts on estrogenic chemicals by stimulating the downstream signaling pathways of the ovary to participate in follicle genesis, growth, and maturation $(28,35)$. It has also been demonstrated that ESR1 mutations have a strong association with POF development (36).

AR is primarily found in the granulosa cells and oocytes of the ovary and is involved in maintaining female fertility by optimizing follicular growth, final follicle development, and ovulation (37). From the results of AR-deficient female mice, a loss of AR protein showed an early decrease in follicle numbers and increased atresia resulting in POF development, indicating that AR may influence follicle atresia by affecting somatic cells apoptosis or oocyte degeneration (38). SRD5A2 is reported to affect steroid hormones by conversion of testosterone to dihydrotestosterone, a more potent androgen than testosterone. It has also been demonstrated that SRD5A2 plays a key role in androgen metabolism (39).

A total of 56 overlapped predicted targets have been found to be associated with the ZSYT pills in the POF treatment. These target points may be enriched by hormone metabolic and biosynthetic processes and chemical synaptic transmission. Most of the enriched signaling pathways were associated with neuroactive ligand-receptor interaction, steroid hormone biosynthesis, and ovarian steroidogenesis.

Among the 56 overlapped predicted targets, 15 genes are involved in neuroactive ligand-receptor interaction. Most of the 15 genes related to the rhodopsin-like receptors, one of the largest families in the G-protein-coupled receptors (GPCRs). These rhodopsin-like GPCRs include hormones, neuropeptides, neurotransmitters, and light receptors, transducing extracellular signals into specifically targeted cells (40). This predicted result indicated that ZSYT pills may affect POF by regulating GPCR-mediated cellular activities.

Three classes of steroid hormones-estrogens, progesterone, and androgens-are regarded as part of the ovary reproductive unit. These steroid hormones can be synthesized by the enzymes in the distinctive steroid-producing cells of the ovary. The enzymes are located in the mitochondria and endoplasmic reticulum and include five hydroxylases, two dehydrogenases, a reductase, and an aromatase (41). Among these important enzymes, $17 \beta-$ HSD and CYP19 were predicted in our KEGG enrichment results. $17 \beta$-HSD converts androstenedione to testosterone and estrone to E2 in granulosa cells. In the presence of CYP19, the converted testosterone could transform to E2 in the granulosa cells and then release into circulation for further maturation and maintenance of the reproductive system. 
Granulosa and theca cells work together to organize the biosynthesis of ovarian steroids. In the results of the predicted pathway, six potential targets, INSR, HSD17B2, AKR1C3 (HSD17B5), CYP19A1, CYP17A1, and IGF1R may be involved. INSR and IGF1R are important for follicular development, ovulation, and luteinization, as demonstrated by the conditional knockout of INSR and IGF1R mice (42). CYP17A1 works only in theca cells to transform pregnenolone to androgens, providing the substrate for CYP19 to convert to estrogens (41). HSD17B2, AKR1C3 (HSD17B5), and CYP19A1 are involved not only in steroid hormone biosynthesis but also in ovarian steroidogenesis.

Because estrogen is essential for reproduction, estrogen therapy has become the mainstay of POF treatment (43). However, limited data are available on the efficacy and safety of long-term use.

Therefore, our findings suggest that the ZSYT pills treat POF primarily by regulating neuroactive ligandreceptor interactions, steroid hormone biosynthesis, and ovarian steroidogenesis resulting in improved levels of E2 and clinical symptoms of the hormone disorders in patients with POF $(11,12)$.

\section{Conclusions}

In conclusion, we have demonstrated that ZSYT pills treat POF primarily by regulating neuroactive ligandreceptor interactions, steroid hormone biosynthesis, and ovarian steroidogenesis. Our study suggests that combining the absorbed components of ZSYT pills in vivo in the POF models and network pharmacology prediction may offer a tool to characterize the mechanism of ZSYT pills in the POF. However, our study has limitations since it has not been validated by additional animal experiments and clinical trials. The details of pharmacological mechanisms of ZSYT pills in treating POF will be investigated in a future study.

\section{Abbreviations}




\begin{tabular}{|c|c|}
\hline Abbreviation & Explaination \\
\hline AKR & Aldo-Keto Reductase Family \\
\hline AKR1C3 & Aldo-Keto Reductase Family 1 Member C3 \\
\hline $\mathrm{AMH}$ & anti-Müllerian hormone \\
\hline AR & Androgen Receptor \\
\hline BP & Biological processes \\
\hline CC & Cellular components \\
\hline CMC-Na & Sodium Carboxymethyl Cellulose \\
\hline CYP17A1 & Cytochrome P450 Family 17 Subfamily A Member 1 \\
\hline CYP19 & Cytochrome P450 Family 19 \\
\hline CYP19A1 & Cytochrome P450 Family 19 Subfamily A Member 1 \\
\hline ESI & Electrospray ionization \\
\hline ESR1 & Estrogen Receptor 1 \\
\hline E2 & Estradiol \\
\hline FSH & Follicle-stimulating hormone \\
\hline GAD & Genetic Association Database \\
\hline GO & Go enrichment analysis gene ontology \\
\hline GPCRs & G-protein-coupled receptors \\
\hline HSD17B2 & Hydroxysteroid 17-Beta Dehydrogenase 2 \\
\hline HSD17B5 & $17 \beta$-hydroxysteroid dehydrogenase type 5 \\
\hline IGF1R & Insulin Like Growth Factor 1 Receptor \\
\hline INSR & Insulin Receptor \\
\hline IVF & in vitro fertilization \\
\hline KEGG & Kyoto Encyclopedia of Genes and Genomes \\
\hline LH & Luteinizing hormone \\
\hline MF & Molecular functions \\
\hline OMIM & Online Mendelian Inheritance in Man Database \\
\hline PharmGkb & Pharmacogenomics Knowledge Base \\
\hline POF & Premature ovarian failure \\
\hline
\end{tabular}




\begin{tabular}{|ll|}
\hline PPI & Protein-protein interaction \\
\hline TCM & Steroid 5 Alpha-Reductase 2 \\
\hline TCMSP & Traditional Chinese medicine \\
\hline TTD & Traditional Chinese Medicine Systems Pharmacology Database \\
\hline $\begin{array}{l}\text { UHPLC-Q-TOF- } \\
\text { MS }\end{array}$ & $\begin{array}{l}\text { Ultra-high performance liquid chromatography-quadrupole time-of-flight mass } \\
\text { spectrometry }\end{array}$ \\
\hline ZSYT & Zishen Yutai \\
\hline 17ß-HSD & $17 \beta$-hydroxysteroid dehydrogenase \\
\hline
\end{tabular}

\section{Declarations}

\section{Ethics approval and consent to participate}

All procedures were conducted in accordance with relevant guidelines and regulations. All methods were reported in accordance with ARRIVE guidelines for the reporting of animal experiments and the experimental protocols were approved by the the Institutional Animal Ethics Committee of the Guangzhou General Pharmaceutical Research Institute Co., Ltd. (IA-PD2021003-01).

\section{Consent for publication}

Not applicable.

\section{Availability of data and materials}

The dataset(s) supporting the conclusions of this article is(are) included within the article (and its additional file(s)).

\section{Competing interests}

The authors declare that the research was conducted in the absence of any commercial or financial relationships that could be construed as a potential conflict of interest.

\section{Funding}

This study was supported by the National Natural Science Foundation of China (Grant No. 82002768 ) and the Science and Technology Program of Guangzhou (Grant No.201807010044).

\section{Authors' contributions}


Shixuan Wang and Yan Li supervised the whole project. Lei Dang performed the major research and wrote the manuscript. Chunbo Zhang, Biru Su, Ning Na, Qiuling Huang, Su Zhou, and Meng Wu provided the technical support. Wenqing Ma, Man Wang, and Pengfei Cui provided their professional expertise.

\section{Acknowledgements}

Not applicable.

\section{References}

1. Kasteren, Y. M. v. Premature ovarian failure. Nederlands tijdschrift voor geneeskunde 2000; 144:2380.

2. Yoldemir, T., Garibova, N. \& Atasayan, K. The association between sexual dysfunction and metabolic syndrome among Turkish postmenopausal women. Climacteric 2019; 22:472-477. doi: 10.1080/13697137.2019.1580256.

3. Towner, M. C., Nenko, I. \& Walton, S. E. Why do women stop reproducing before menopause? A lifehistory approach to age at last birth. Philosophical transactions of the Royal Society of London. Series B, Biological sciences 2016; 371. doi: 10.1098/rstb.2015.0147.

4. Sheikhansari, G., Aghebati-Maleki, L., Nouri, M., Jadidi-Niaragh, F. \& Yousefi, M. Current approaches for the treatment of premature ovarian failure with stem cell therapy. Biomedicine \& pharmacotherapy 2018; 9. doi: 10.1016/j.biopha.2018.03.056.

5. Coulam, C. B., Adamson, S. C. \& Annegers, J. F. Incidence of premature ovarian failure. Obstetrics and gynecology 1986; 67:604-606.

6. Santoro, N. Mechanisms of premature ovarian failure. Annales d'endocrinologie 2003; 64: 87-92.

7. Ghahremani-Nasab, M., Ghanbari, E., Jahanbani, Y., Mehdizadeh, A. \& Yousefi, M. Premature ovarian failure and tissue engineering. Journal of cellular physiology 2020; 235: 4217-4226. doi: 10.1002/jcp.29376.

8. Lin, J. Li, X.-L., Song, H., Li, Q., Wang, M.-Y., Qiu, X.-M., Li, D.-J. \& Wang, L. A general description for Chinese medicine in treating premature ovarian failure. Chinese journal of integrative medicine 2017; 23:91-97. doi: 10.1007/s11655-016-2642-7.

9. Zhang, X.-L. \& Zhao, X.-Y. Regulatory Effect of Zishen Yutai Pill on the Biological Behavior of Oocytes in Immune Infertility Model Rats. Chinese journal of integrated traditional and Western medicine 2017; 37:351-355.

10. Zhang, Y., Yan, W., Ge, P.-F., Li, Y. \& Ye, Q. Study on prevention effect of Zishen Yutai pill combined with progesterone for threatened abortion in rats. Asian Pacific journal of tropical medicine 2016; 9:577-581. doi: 10.1016/j.apjtm.2016.04.002.

11. Jie, J. \& Chen, M. Clinical observation of Zishen Yutai pill combined with Kelingmeng in the treatment of premature ovarian failure. Journal of Hunan University of Chinese Medicine 2017; 37. doi:10.3969/j.issn.1674-070X.2017.12.023. 
12. Pang, Z., Liang, Q., Zhong, X., Peng, J. \& Li, Y. The Clinical Research on Zishen Yutai Pill in the Treatment of Diminished Ovarian Reserve for 300 Cases. Chinese Medicine Modern Distance Education of China 2017; 15.

13. Cao, J. -L., Lei, T., Wu, S.-J., Li, H.-Y., Lin, R.-Z., Ning, N., Geng, C.-X., Wang, S.-P., Wu, X, Li, P. \& Wang, Y.T. Development of a comprehensive method combining UHPLC-CAD fingerprint, multi-components quantitative analysis for quality evaluation of Zishen Yutai Pills: A step towards quality control of Chinese patent medicine. Journal of pharmaceutical and biomedical analysis 2020; 191. doi: 10.1016/j.jpba.2020.113570.

14. Deng, Y.-J., Gao, X., Feng, T.-T., Wang, Z.-Z., Xiao, W., Xiong, Z.-L. \& Zhao, L.-S. Systematically characterized mechanism of treatment for lumbar disc herniation based on Yaobitong capsule ingredient analysis in rat plasma and its network pharmacology strategy by UPLC-MS/MS. Journal of ethnopharmacology 2020; 260: 113097. doi: 10.1016/j.jep.2020.113097.

15. Liu, Y., Zhang, G.-J., Sun, S.-Q. \& Noda, I. Study on similar traditional Chinese medicines Cornu Cervi Pantotrichum, Cornu Cervi and Cornu Cervi Degelatinatum by FT-IR and 2D-IR correlation spectroscopy. Journal of pharmaceutical and biomedical analysis 2010; 52:631-635. doi: 10.1016/j.jpba.2010.01.045.

16. Luo, J. , Zhang,X.-Y., Wang, Y.-Y., Yang, J., Fan, Z.-H. \& Yu, L. -M. Difference of degree of premature ovarian failure induced by cisplatin in mice. Journal of Zunyi Medical College 2013; 36.

17. Lee, E.-H, Han, S.-E., Park, M.-J., Kim, H.-J., Kim, H.-G., Kim, C.-W., Joo, B.-S. \& Lee, K. -S. Establishment of Effective Mouse Model of Premature Ovarian Failure Considering Treatment Duration of Anticancer Drugs and Natural Recovery Time. Journal of menopausal medicine 2018; 24:196-203. doi: $10.6118 / \mathrm{jmm} .2018 .24 .3 .196$.

18. Wu, Y., Liu, W. \& Zhao, W. The contribution of serum pharmacology to pharmacology, pharmacodynamics and new drug development. Chinese Tissue Engineering Research 2018; 22.

19. Zhang, Y. , Gao, J., Zhang, Q., Wang, K., Yao, W.-F., Bao, B.-H., Zhang, L. \& Tang Y.-P. Interpretation of Euphorbia Kansui Stir-Fried with Vinegar Treating Malignant Ascites by a UPLC-Q-TOF/MS Based Rat Serum and Urine Metabolomics Strategy Coupled with Network Pharmacology. Moleucles 2018; 23. doi: $10.3390 /$ molecules23123246.

20. Zhang, J.-K., Yu, Q.-H., Cheng, H.-Y., Ge, Y.-Q., Liu, H., Ye, X.-Q. \& Chen, Y. Metabolomic Approach for the Authentication of Berry Fruit Juice by Liquid Chromatography Quadrupole Time-of-Flight Mass Spectrometry Coupled to Chemometrics. Journal of agricultural and food chemistry $2018 ; 66: 8199-$ 8208. doi: $10.1021 /$ acs.jafc.8b01682.

21. Hiebert, L. M., Wice, S. M. \& Abdelhameed, T. Evidence for the absorption of heparin by rat stomach. Biomedicine \& pharmacotherapy 2007; 61:68-74. doi: 10.1016/j.biopha.2006.08.006.

22. Mering, C. v., Huynen, M., Jaeggi, D., Schmidt, S., Bork, P. \& Snel, B. STRING: a database of predicted functional associations between proteins. Nucleic acids research 2003; 31:258-261. doi: 10.1093/nar/gkg034. 
23. Huang, D. W., Sherman, B. T. \& Lempicki, R. A. Systematic and integrative analysis of large gene lists using DAVID bioinformatics resources. Nature protocols 2009; 4:44-57. doi: 10.1038/nprot.2008.211.

24. Smoot, M. E., Ono, K., Ruscheinski, J., Wang, P.-L. \& Ideker, T. Cytoscape 2.8: new features for data integration and network visualization. Bioinformatics 2011;27:431-432. doi: 10.1093/bioinformatics/btq675.

25. Ma, J. X., Wang, B., Li, H.S., Yu, J. \& Chen, W.Q. Uncovering the mechanisms of leech and centipede granules in the treatment of diabetes mellitus-induced erectile dysfunction utilising network pharmacology. Journal of ethnopharmacology 2021; 265. doi: 10.1016/j.jep.2020.113358.

26. Maharajan, K., Xia, Q., Duan, X.-Y., Tu, P.-F., Zhang, Y. \& Liu, K.-C. Therapeutic importance of Zishen Yutai Pill on the female reproductive health: A review. J Ethnopharmacol 2021; 281, 2021 Dec 2025. doi: 10.1016/j.jep.2021.114523.

27. Luisi, S. , Orlandini.C., Regini, C., Pizzo, A., Vellucci, F. \& Petraglia F. Premature ovarian insufficiency: from pathogenesis to clinical management. J Endocrinol Invest 2015; 38:597-603. doi: 10.1007/s40618-014-0231-1.

28. Patel, S., Zhou, C., Rattan, S. \& Flaws, J. A. Effects of Endocrine-Disrupting Chemicals on the Ovary. Biol Reprod 2015; 93. doi: 10.1095/biolreprod.115.130336.

29. Hanukoglu, I. Steroidogenic enzymes: structure, function, and role in regulation of steroid hormone biosynthesis. J Steroid Biochem Mol Biol 1992; 43:779-804. doi: 10.1016/0960-0760(92)90307-5.

30. Karman, B. N., Basavarajappa, M. S., Hannon, P. \& Flaws, J. A. Dioxin exposure reduces the steroidogenic capacity of mouse antral follicles mainly at the level of HSD17B1 without altering atresia. Toxicol Appl Pharmacol 2012; 264:1-12. doi: 10.1016/j.taap.2012.07.031.

31. Jin, Y. \& Penning, T. M. Aldo-keto reductases and bioactivation/detoxication. Annu Rev Pharmacol Toxicol 2007; 47:263-292. doi: 10.1146/annurev.pharmtox.47.120505.105337.

32. Bray, J. E., Marsden, B. D. \& Oppermann, U. The human short-chain dehydrogenase/reductase (SDR) superfamily: a bioinformatics summary. Chem Biol Interact 2009; 178:99-109. doi: 10.1016/j.cbi.2008.10.058.

33. Penning, T. M. AKR1C3 (type 5 17ß-hydroxysteroid dehydrogenase/prostaglandin F synthase): Roles in malignancy and endocrine disorders. Mol Cell Endocrinol 2019; 489:82-91. doi: 10.1016/j.mce.2018.07.002.

34. Yepuru, M. , Wu, Z.Z., Kulkarni, A., Yin, F., Barret, C.M., Kim, J., Steiner, M.S., Miller, D.D., Dalton, J.T. \& Narayanan, R. Steroidogenic enzyme AKR1C3 is a novel androgen receptor-selective coactivator that promotes prostate cancer growth. Clin Cancer Res 2013; 19:5613-5625. doi: 10.1158/10780432.CCR-13-1151.

35. Craig, Z. R., Wang, W. \& Flaws, J. A. Endocrine-disrupting chemicals in ovarian function: effects on steroidogenesis, metabolism and nuclear receptor signaling. Reproduction 2011; 142:633-646. doi:10.1530/REP-11-0136.

36. Qin, Y. -Y., Sun, M., You, L., Wei, D.-Y., Sun, J.-L., Liang, X.-Y., Zhang, B., Jiang, H., Xu, J.-F. \& Chen, Z.-J. ESR1, HK3 and BRSK1 gene variants are associated with both age at natural menopause and 
premature ovarian failure. Orphanet J Rare Dis 2012; 7. doi: 10.1186/1750-1172-7-5.

37. Walters, K. A., Simanainen, U. \& Handelsman, D. J. Molecular insights into androgen actions in male and female reproductive function from androgen receptor knockout models. Hum Reprod Update 2010; 16:543-558. doi: 10.1093/humupd/dmq003.

38. Shiina, H., Matsumoto, T., Sato, T., Igarashi, K., Miyamoto, J., Takemasa, S., Sakari, M., Takada, I., Nakamura, T., Metzger, D., Chambon, P., Kanno, J., Yoshikawa, H. \& Kato, S. Premature ovarian failure in androgen receptor-deficient mice. Proc Natl Acade Sci U S A 2006; 103:224-229. doi:

10.1073/pnas.0506736102.

39. Beesley, J. , Jordan, S.-J., Spurdle, A.-B., Song, H.-L., Ramus, S.-J., Kjaer, S.-K., Hogdall, E., DiCioccio, R.-A., McGuire, V., Whittemore, A.-S., Gayther, S.-A., Pharoah, P.D.-P., Webb, P.-M., Chenevix-Trench, G., Australian Ovarian Cancer Study Group, Australia Cancer Study (Ovarian Cancer) \& Australian Breast Cancer Family Study. Association between single-nucleotide polymorphisms in hormone metabolism and DNA repair genes and epithelial ovarian cancer: results from two Australian studies and an additional validation set. Cancer Epidemiol Biomarkers Prev 2007; 16:2557-2265. doi: 10.1158/10559965.EPI-07-0542.

40. Casey, P. J. \& Gilman, A. G. G protein involvement in receptor-effector coupling. J Biol Chem 1988; 263:2577-2580.

41. Gupta, M. K. \& Chia, S.-Y. Clinical Reproductive Medicine and Surgery 2013.

42. Sekulovski, N., Whorton, A. E., Shi, M., Hayashi, K. \& 2nd, J. A. M. Periovulatory insulin signaling is essential for ovulation, granulosa cell differentiation, and female fertility. FASEB J 2020; 34:23762391, doi: 10.1096/fj.201901791R.

43. Practice, C. o. G. Hormone Therapy in Primary Ovarian Insufficiency. Obstet Gynecol 2017; 129. doi: 10.1097/AOG.0000000000002040.

\section{Tables}

Table 1 POF-related target genes interacting with absorbed compounds from ZSYT pills. 


\section{No. Symbol Gene Name Name}

\begin{tabular}{lll}
$\mathbf{1}$ & ABCB1 & ATP Binding Cassette Subfamily B Member 1 \\
\hline $\mathbf{2}$ & ACE & Angiotensin I Converting Enzyme \\
\hline $\mathbf{3}$ & ACVR2B & Activin A Receptor Type 2B \\
\hline $\mathbf{4}$ & AKR1C2 & Aldo-Keto Reductase Family 1 Member C2 \\
\hline $\mathbf{5}$ & AKR1C3 & Aldo-Keto Reductase Family 1 Member C3 \\
\hline $\mathbf{6}$ & APBA3 & Amyloid Beta Precursor Protein Binding Family A Member 3 \\
\hline $\mathbf{7}$ & AR & Androgen Receptor \\
\hline $\mathbf{8}$ & CCR3 & C-C Motif Chemokine Receptor 3 \\
\hline $\mathbf{9}$ & CHFR & Checkpoint With Forkhead And Ring Finger Domains \\
\hline $\mathbf{1 0}$ & CHRNA4 & Cholinergic Receptor Nicotinic Alpha 4 Subunit \\
\hline $\mathbf{1 1}$ & CHRNA7 & Cholinergic Receptor Nicotinic Alpha 7 Subunit \\
\hline $\mathbf{1 2}$ & CITED2 & $\begin{array}{l}\text { Cbp/P300 Interacting Transactivator With Glu/Asp Rich Carboxy-Terminal } \\
\text { Domain 2 }\end{array}$ \\
\hline $\mathbf{1 3}$ & CNR1 & Cannabinoid Receptor 1 \\
\hline $\mathbf{1 4}$ & CRHR1 & Corticotropin Releasing Hormone Receptor 1 \\
\hline $\mathbf{1 5}$ & CYP17A1 & Cytochrome P450 Family 17 Subfamily A Member 1 \\
\hline $\mathbf{1 6}$ & CYP19A1 & Cytochrome P450 Family 19 Subfamily A Member 1 \\
\hline $\mathbf{1 7}$ & CYP2C19 & Cytochrome P450 Family 2 Subfamily C Member 19 \\
\hline $\mathbf{1 8}$ & DRD2 & Dopamine Receptor D2 \\
\hline $\mathbf{1 9}$ & DRD3 & \begin{tabular}{l} 
Dopamine Receptor D3 \\
\hline
\end{tabular} \\
\hline
\end{tabular}


20 ESR1 Estrogen Receptor 1

21 ESR2 Estrogen Receptor 2

22 F2 Coagulation Factor II, Thrombin

23 FGFR1 Fibroblast Growth Factor Receptor 1

24 GABBR2 Gamma-Aminobutyric Acid Type B Receptor Subunit 2

25 GSTT1 Glutathione S-Transferase Theta 1

26 HSD17B2 Hydroxysteroid 17-Beta Dehydrogenase 2

27 HSD17B3 Hydroxysteroid 17-Beta Dehydrogenase 3

28 HSD17B4 Hydroxysteroid 17-Beta Dehydrogenase 4

29 HTR1A 5-Hydroxytryptamine Receptor 1A

30 HTR2A 5-Hydroxytryptamine Receptor 2A

31 IGF1R Insulin Like Growth Factor 1 Receptor

32 IGFBP2 Insulin Like Growth Factor Binding Protein 2

33 IL6 Interleukin 6

$34 \quad$ INSR Insulin Receptor

35 MAOA Monoamine Oxidase A

36 MAOB Monoamine Oxidase B

37 MAPK14 Mitogen-Activated Protein Kinase 14

38 NR3C1 Nuclear Receptor Subfamily 3 Group C Member 1

39 OPRD1 $\quad$ Opioid Receptor Delta 1

40 OPRK1 $\quad$ Opioid Receptor Kappa 1 


\begin{tabular}{lll}
$\mathbf{4 1}$ & OPRM1 & Opioid Receptor Mu 1 \\
\hline $\mathbf{4 2}$ & PCSK9 & Proprotein Convertase Subtilisin/Kexin Type 9 \\
\hline $\mathbf{4 3}$ & PGR & Progesterone Receptor \\
\hline $\mathbf{4 4}$ & PPARG & Peroxisome Proliferator Activated Receptor Gamma \\
\hline $\mathbf{4 5}$ & PTPN11 & Protein Tyrosine Phosphatase Non-Receptor Type 11 \\
\hline $\mathbf{4 6}$ & SERPINE1 & Serpin Family E Member 1 \\
\hline $\mathbf{4 7}$ & SHBG & Sex Hormone Binding Globulin \\
\hline $\mathbf{4 8}$ & SLC6A3 & Solute Carrier Family 6 Member 3 \\
\hline $\mathbf{4 9}$ & SLC6A4 & Solute Carrier Family 6 Member 4 \\
\hline $\mathbf{5 0}$ & SRD5A1 & Steroid 5 Alpha-Reductase 1 \\
\hline $\mathbf{5 1}$ & SRD5A2 & Steroid 5 Alpha-Reductase 2 \\
\hline $\mathbf{5 2}$ & SREBF2 & Sterol Regulatory Element Binding Transcription Factor 2 \\
\hline $\mathbf{5 3}$ & SSTR3 & Somatostatin Receptor 3 \\
\hline $\mathbf{5 4}$ & TNF & Tumor Necrosis Factor \\
\hline $\mathbf{5 5}$ & UGT2B7 & UDP Glucuronosyltransferase Family 2 Member B7 \\
\hline $\mathbf{5 6}$ & VDR & Vitamin D Receptor \\
\hline
\end{tabular}

\section{Figures}

\section{Figure 1}

Schematics of the process for identifying absorbed components of ZSYT pills in the POF mouse serum and determining 517 their targets of activity against POF via integrated UHPLC-Q-TOF-MS analysis and network pharmacology approach. 
A

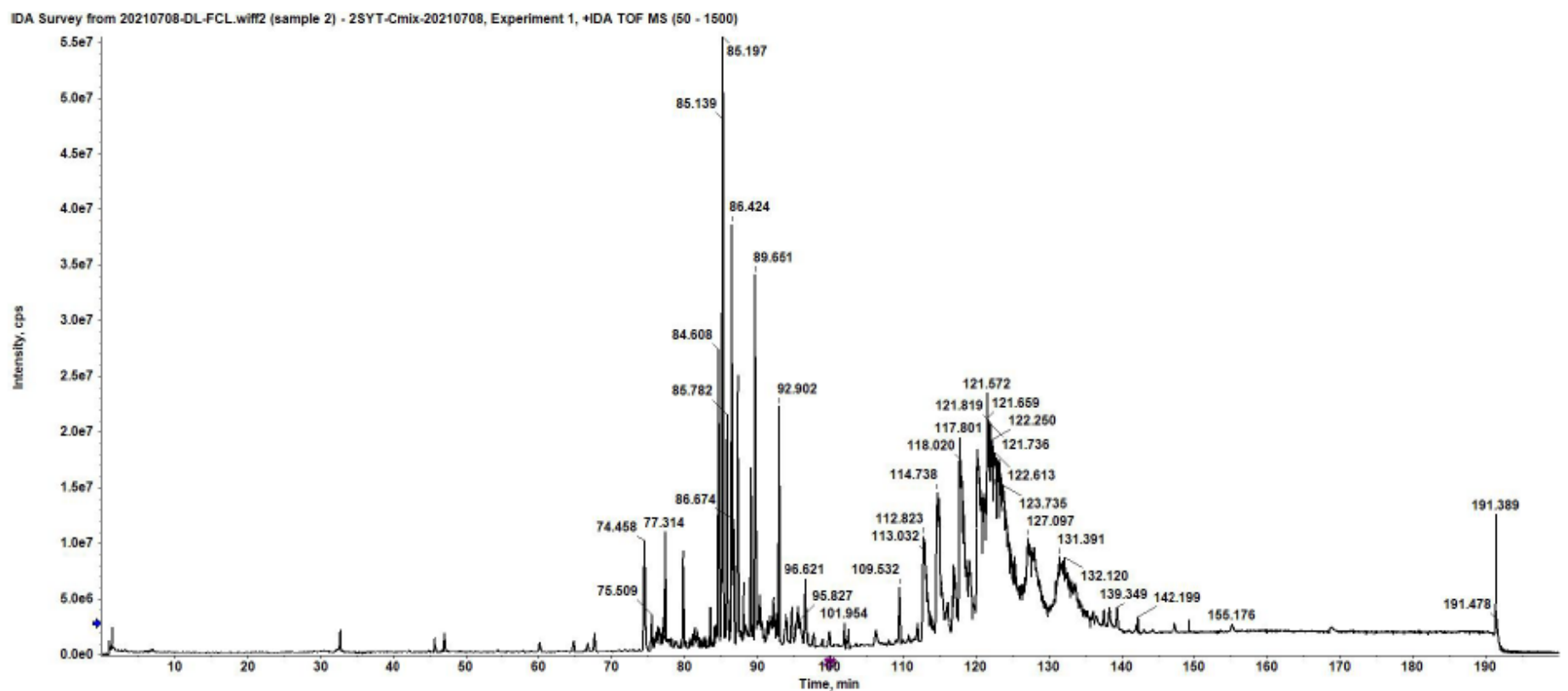

B

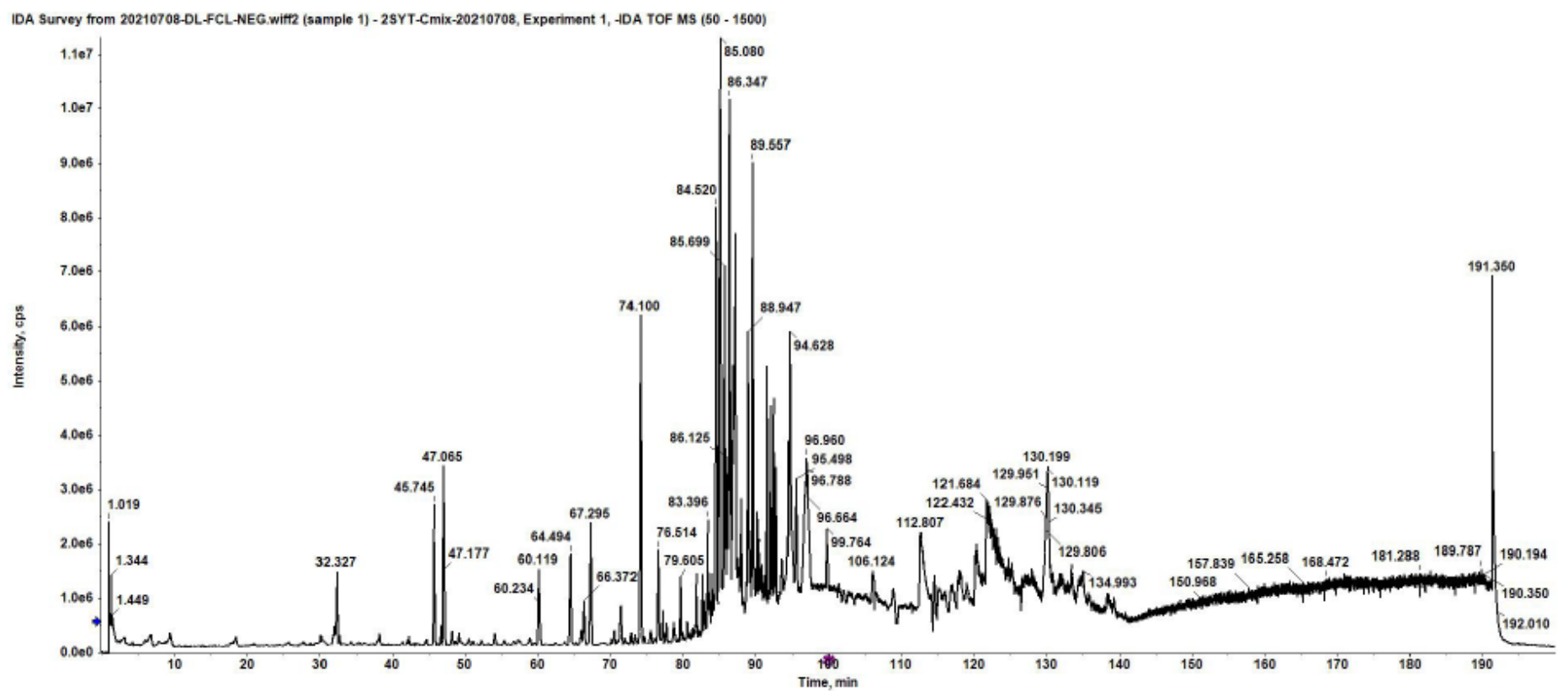

Figure 2

Total ion flow diagram of the absorbed components in serum after the oral administration of ZSYT pills in POF mice analyzed via UHPLC-Q-TOF-MS. (A) Positive ion mode. (B) Negative ion mode.

Figure 3 
The compound-target network for components absorbed from ZSYT pills administered orally in a POF mouse model.

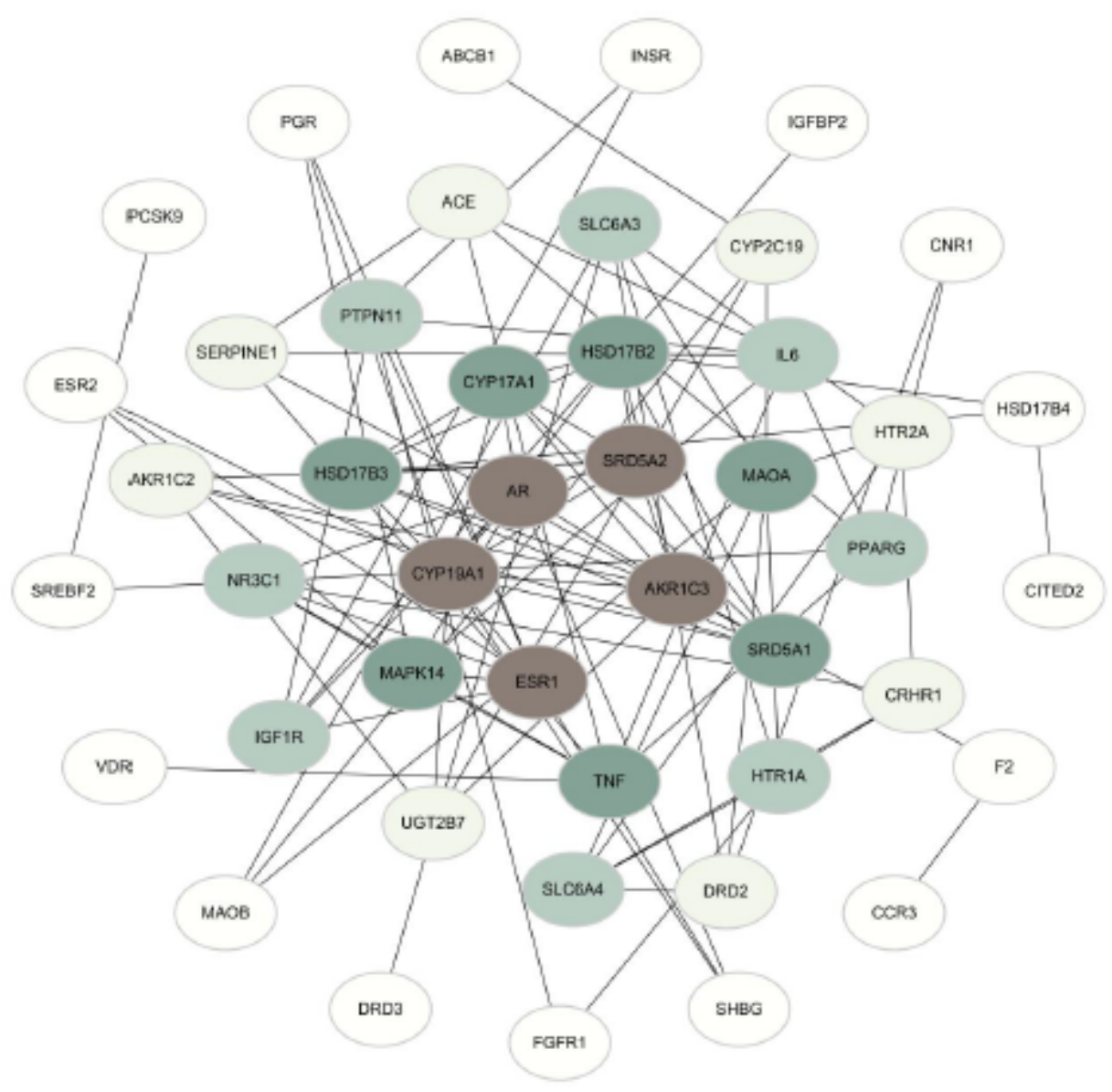

\section{Figure 4}

The ZSYT absorbed components- POF PPI network.

\section{Figure 5}

GO enrichment analysis of therapeutic targets of absorbed components of ZSYT pills on POF. (A) Biological process. (B) Cellular components. (C) Molecular functions 


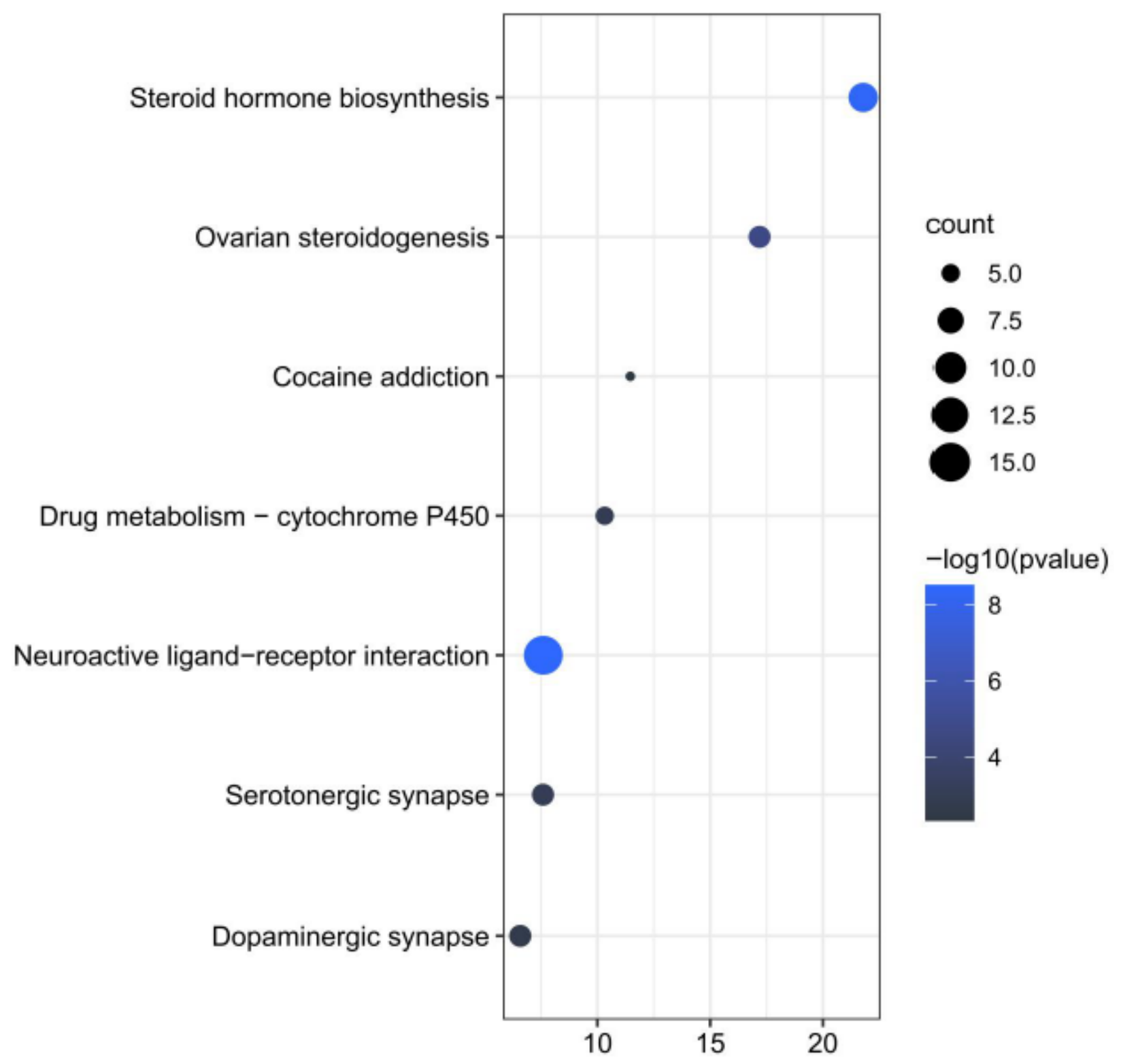

Figure 6

KEGG pathway enrichment analysis of potential targets of orally 555 absorbed ZSYT pills components from in POF.

\section{Supplementary Files}

This is a list of supplementary files associated with this preprint. Click to download.

- SupplementaryMaterial20211207.pdf 